\title{
Action growth for black holes in modified gravity
}

\author{
Lorenzo Sebastiani, ${ }^{1,2, *}$ Luciano Vanzo, ${ }^{1,2, \dagger}$ and Sergio Zerbini ${ }^{1,2, *}$ \\ ${ }^{1}$ Dipartimento di Fisica, Università di Trento, Via Sommarive 14, 38123 Povo, Trento, Italy \\ ${ }^{2}$ Trento Institute for Fundamental Physics and Applications (TIFPA), INFN, \\ Via Sommarive 14, 38123 Povo, Trento, Italy
}

(Received 18 October 2017; published 6 February 2018)

\begin{abstract}
The general form of the action growth for a large class of static black hole solutions in modified gravity which includes $F(R)$-gravity models is computed. The cases of black hole solutions with nonconstant Ricci scalar are also considered, generalizing the results previously found and valid only for black holes with constant Ricci scalar. An argument is put forward to provide a physical interpretation of the results, which seem tightly connected with the generalized second law of black hole thermodynamics.
\end{abstract}

DOI: 10.1103/PhysRevD.97.044009

\section{INTRODUCTION}

Recently, Brown et al. proposed an interesting conjecture in the AdS/CFT framework, according to which the quantum computational complexity of a holographic state may be inferred from the classical action related to a specific region in the bulk [1]. Such a proposal has been checked in the context of the anti-de Sitter (AdS) black holes (BHs) in general relativity (GR), and this is an interesting test for the complexity/action (CA) duality [2]. This conjecture is a refined version of a previous one which states that the complexity is dual to the spatial volume of a maximal slice behind the horizon [3]. Since the properties of the black hole interior are represented on the holographic boundary, it is possible to find the boundary state by computing the classical action of the space-time region inside the $\mathrm{BH}$ (in the so-called "Wheeler-DeWitt" (WdW) patch; see Ref. [4] for a detailed geometrical analysis of the issue). After calculating the growth of the complexity at the late time, it is found that in the case of neutral black holes the action growth is bounded by a term proportional to the $\mathrm{BH}$ energy.

In modified theories of gravity several attempts have been made in order to calculate the action growth for neutral and charged AdS black holes; see for example Refs. [5-7].

In this paper, our aim is to investigate the action growth in the case of the black holes within a class of modified gravity. We are mainly interested in $F(R)$ theories of gravity, where the action is given by a general function

\footnotetext{
*lorenzo.sebastiani@unitn.it

†uciano.vanzo@sunitn.it

zerbini@science.unitn.it
}

Published by the American Physical Society under the terms of the Creative Commons Attribution 4.0 International license. Further distribution of this work must maintain attribution to the author(s) and the published article's title, journal citation, and DOI. Funded by SCOAP. of the Ricci scalar $R$. Such models represent the simplest generalization of Einstein's theory, and, in general, they admit the existence of Schwarzschild dS/AdS black holes, namely solutions with constant Ricci curvature. Beside these "trivial" black hole solutions, we present the computation of the action growth associated with nontrivial black hole (vacuum) solutions with nonconstant Ricci curvature, found in Refs. [8-13]. Some of these static solutions represent "dirty BHs" [14], namely ones in which the (00)- and (11)-metric components are related as $g_{00} g_{11} \neq-1$. They typically involve scalar hairs.

The thermodynamical interpretation for such BHs solutions is still an open issue (see for instance Refs. [15-19]) and the relation between the action growth and the $\mathrm{BH}$ energy in $F(R)$ gravity should be careful considered. For our purposes, we make use of the fact that in most cases and within $F(R)$ gravity, the $\mathrm{BH}$ energy may be obtained by deriving the first law of $\mathrm{BH}$ thermodynamics from the equations of motion [20]. In fact, when only one integration constant appears in the solution, it is possible to identify it with the Killing energy of the black hole itself.

For the black holes with constant Ricci curvature, we confirm the results previously obtained. For dirty black holes with nonconstant Ricci scalar, the so-called KodamaHayward energy appears in the action growth. Finally, we also investigate the action growth for a modified gravity model with an additional term based on the Weyl tensor, which is not belonging to the $F(R)$ class.

Quite apart from computations, it is also important to assess the validity of the conditions allowing us to restrict attention to spherically symmetric solutions beyond the obvious demand of simplicity and the advantage of working with exact solutions, and to relate the action grow with the physics of black hole evaporation. In this context, the more important property is the grows being proportional to the internal energy of the black hole. We show that this is equivalent, for neutral nonrotating black holes, to the 
simultaneous validity of the generalized second law together with the Pendry's inequality [21] characterizing the information rate of a single communication channel, whose exact definition in general depends on the physical character of the information carriers and the medium by which they propagate. ${ }^{1}$ We recall that the generalized second law stipulates that the entropy of the black hole plus the one carried away by the Hawking radiation should satisfy the inequality

$$
0 \leq \dot{S}_{\mathrm{BH}}+\dot{S}_{\mathrm{rad}},
$$

where the dot denotes the time derivative, ${ }^{2}$ while what Pendry says (adapted in a form suitable to us) is that for a channel fed by power $P$, we have

$$
\dot{S}_{+} \leq\left(\frac{\pi P}{3}\right)^{1 / 2}
$$

where $\dot{S}_{+}$is the entropy flow along the channel. Identifying $S_{+}$with $S_{\mathrm{rad}}$ and $P=-\dot{E}_{\mathrm{BH}}$, both are satisfied by the black holes and together would imply that the action grow scales with the internal energy, so we may say that the neutral black hole is a kind of one-dimensional information channel in the sense specified by Pendry, as was shown long ago by Bekenstein by other means [23]. Adopting the CA conjecture, one may conclude that the rate of complexity grow of (the boundary horizon state) of the black hole cannot be more than twice its thermodynamical energy.

The rest of the paper is organized in the following way. In Sec. II, the equations of motion for static spherical symmetric (SSS) metric of $F(R)$ gravity are calculated, starting from a suitable action in which the associated boundary term has been taken into account. In Sec. III we present a derivation of the first law of $\mathrm{BH}$ thermodynamics which allows us to obtain the BH Killing energy in the framework of $F(R)$ gravity. In Sec. IV the general formalism for the evaluation of the action growth in $F(R)$ gravity is presented and applied to the black holes previously introduced. We use a simpler approach making full use of the assumed spherical symmetry. For a full treatment in general relativity in anti-de Sitter space, see the recent comprehensive paper of D. Carmi et al. [24]. Section $\mathrm{V}$ is devoted to the calculation of the action growth for a BH solution in a Weyl model of modified gravity. After these rather technical sections, in Sec. VI we give a physical discussion of the results thereby obtained. The conclusions and final remarks are given in Sec. VII, while in the appendixes the explicit calculations of the boundary terms of the action in $F(R)$ and Weyl gravity are presented.

In this work we use units of $k_{\mathrm{B}}=c=\hbar=1$.

\footnotetext{
${ }^{1}$ We are using these terms in the sense of Shannon's communication theory [22]. One may conveniently think of a onedimensional channel as an optical fiber.

${ }^{2}$ Here time derivatives are taken with respect to retarded coordinate time, or equivalently to time at infinity.
}

\section{ACTION AND EQUATIONS OF MOTION IN $\boldsymbol{F}(R)$ GRAVITY}

To begin with, we recall that the action for a generic modified gravity model depending only on the scalar Ricci curvature in the vacuum and in four dimensions may be written as (see for example [25-27])

$$
I=\int_{\mathcal{M}} d^{4} x \sqrt{-g} F(R)
$$

where $\mathcal{M}$ is a four-dimensional space-time manifold with boundary $\partial \mathcal{M}, g$ is the determinant of the metric tensor $g_{\mu \nu}\left(x^{\mu}\right)$, and $F(R)$ is a function of the Ricci scalar $R$.

As in GR, in order to deal with a proper well-posed variational problem for the metric tensor [28], one needs to subtract to the Lagrangian a suitable boundary term. In the so-called Jordan frame, one has to work with the following action [29-36],

$\hat{I}=\int_{\mathcal{M}} d^{4} x \sqrt{-g} F(R)-2 \int_{\partial \mathcal{M}} d^{3} x \sqrt{-h} F^{\prime}(R) K$,

where $K$ is the trace of the extrinsic curvature related to $\partial M$ and $h$ is the trace of the three-dimensional induced metric $h_{i j}\left(x^{i}\right)$. Usually the boundary has topology $S^{2} \times R$ and is foliated by two-spheres. The signature can be either timelike or null, but not spacelike. In the null case there are some unresolved ambiguities [4]. If it is not orthogonal to the space-time foliation in the Hamiltonian formulation then suitable bolt terms have to be added, along the lines discussed in [37] in GR, for example. The field equations can be derived and one gets

$F^{\prime}(R) R_{\mu \nu}-\frac{1}{2} F(R) g_{\mu \nu}-\left(\nabla_{\mu} \nabla_{\nu}-g_{\mu \nu} \nabla_{\alpha} \nabla^{\alpha}\right) F^{\prime}(R)=0$,

where $\nabla_{\mu}$ is the covariant derivative associated to the metric tensor $g_{\mu \nu}\left(x^{\mu}\right)$ and the prime denotes the derivative with respect to the Ricci scalar. As is well known, these above sets of differential equations are difficult to solve. However, if one is looking for exact solutions admitting a space-time symmetry, one may proceed via the so-called minisuperspace approach (see for example Refs. [38-40]).

In this paper we consider a class of static spherically symmetric topological space-times defined by the metric

$$
d s^{2}=-\mathrm{e}^{2 \alpha(r)} B(r)+\frac{d r^{2}}{B(r)}+r^{2} d \Omega_{k}^{2},
$$

where $d \Omega_{k}^{2}$ is the metric of a constant curvature compact two-dimensional space, the so-called horizon manifold with areal radius $r$, and admitting three different topologies, namely spherical, flat (toroidal really) or Riemann surfaces, depending on the $k$ parameter, $k=1,0,-1$, respectively. Furthermore, $\alpha(r)$ and $B(r)$ are functions of the radial coordinate only. 
The associated Ricci scalar reads

$$
R=-3\left[\frac{d}{d r} B(r)\right] \frac{d}{d r} \alpha(r)-2 B(r)\left[\frac{d}{d r} \alpha(r)\right]^{2}-\frac{d^{2}}{d r^{2}} B(r)-2 B(r) \frac{d^{2}}{d r^{2}} \alpha(r)-4 \frac{\frac{d}{d r} B(r)}{r}-4 \frac{B(r) \frac{d}{d r} \alpha(r)}{r}-2 \frac{B(r)}{r^{2}}+\frac{2 k}{r^{2}} .
$$

In what follows, we implement the minisuperspace approach following Ref. [12].

First from Appendix A we note that in the case of the metric (6) the related boundary term is a total divergence with respect to $r$ and may be written as

$$
\begin{aligned}
B T & =-V_{k} \int d t \int d r \frac{d}{d r}\left[F^{\prime}(R) \mathrm{e}^{\alpha(r)} r^{2}\left(\frac{d B(r)}{d r}+2 B(r) \frac{d \alpha(r)}{d r}+\frac{4 B(r)}{r}\right)\right], \\
& =-V_{k} \int d t\left[F^{\prime}(R) \mathrm{e}^{\alpha(r)} r^{2}\left(\frac{d B(r)}{d r}+2 B(r) \frac{d \alpha(r)}{d r}+\frac{4 B(r)}{r}\right)\right],
\end{aligned}
$$

where $V_{k}$ is the volume of the horizon manifold, namely $V_{1}=4 \pi$ for the sphere, $V_{0}=\operatorname{Im} \tau$, with $\tau$ being the Teichmüller parameter for the torus, and finally $V_{-1}=4 \pi(g-1), 2<g$, for the compact hyperbolic manifold with genus $g$ [41].

In order to deal with a standard Lagrangian with quantities admitting only first order derivatives with respect to $r$, one may introduce in the action (3) evaluated with respect to the metric (6) a Lagrangian multiplier $\lambda$ in the following way:

$$
\begin{aligned}
I= & \int_{\mathcal{M}} d^{4} x\left(\mathrm{e}^{\alpha(r)} r^{2}\right)\left[F(R)-\lambda\left[R+3\left[\frac{d}{d r} B(r)\right] \frac{d}{d r} \alpha(r)+2 B(r)\left[\frac{d}{d r} \alpha(r)\right]^{2}\right.\right. \\
& \left.\left.+\frac{d^{2}}{d r^{2}} B(r)+2 B(r) \frac{d^{2}}{d r^{2}} \alpha(r)+4 \frac{\frac{d}{d r} B(r)}{r}+4 \frac{B(r) \frac{d}{d r} \alpha(r)}{r}+2 \frac{B(r)}{r^{2}}-\frac{2 k}{r^{2}}\right]\right] .
\end{aligned}
$$

Thus, the variation with respect to $R$ leads to Eq. (7) after the identification

$$
\lambda=F^{\prime}(R) .
$$

Now, integrating by parts, it is possible to write the action in the standard form with respect to the variables $\alpha(r), B(r)$ and $R=R(r)$, namely

$$
\begin{aligned}
I= & V_{k} \int d t \int d r e^{\alpha(r)}\left\{r^{2}\left(F(R)-F^{\prime}(R) R\right)+F^{\prime}(R)\left(2 k+2 r \frac{d B(r)}{d r}+2 B(r)+4 r B(r) \frac{d \alpha(r)}{d r}\right)\right. \\
& \left.+F^{\prime \prime}(R) \frac{d R}{d r} r^{2}\left(\frac{d B(r)}{d r}+2 B(r) \frac{d \alpha(r)}{d r}+\frac{4 B(r)}{r}\right)\right\}+B T,
\end{aligned}
$$

where we take into account the equalities in (8) and (10). As a consequence, one may work only with the new bulk action, obtained subtracting the correct boundary term,

$$
\begin{aligned}
\hat{I}= & V_{k} \int d t \int d r e^{\alpha(r)}\left\{r^{2}\left(F(R)-F^{\prime}(R) R\right)+F^{\prime}(R)\left(2 k+2 r \frac{d B(r)}{d r}+2 B(r)+4 r B(r) \frac{d \alpha(r)}{d r}\right)\right. \\
& \left.+F^{\prime \prime}(R) \frac{d R}{d r} r^{2}\left(\frac{d B(r)}{d r}+2 B(r) \frac{d \alpha(r)}{d r}+\frac{4 B(r)}{r}\right)\right\} .
\end{aligned}
$$

Finally, the equations of motion can be obtained by making the variation with respect to $\alpha(r)$ and $B(r)$ and are given by (see also Appendix B)

$$
\begin{gathered}
V_{k} \mathrm{e}^{\alpha}(r)\left[r^{2}\left(R F^{\prime}(R)-F(R)\right)-2 F^{\prime}(R)\left(k-B(r)-r \frac{d B(r)}{d r}\right)\right. \\
\left.+2 B(r) F^{\prime \prime}(R) r^{2}\left[\frac{d^{2} R}{d r^{2}}+\left(\frac{2}{r}+\frac{d B(r) / d r}{2 B(r)}\right) \frac{d R}{d r}+\frac{F^{\prime \prime \prime}(R)}{F^{\prime \prime}(R)}\left(\frac{d R}{d r}\right)^{2}\right]\right]=0, \\
V_{k} \mathrm{e}^{\alpha}(r)\left[\frac{1}{r^{2}} \frac{d \alpha(r)}{d r}\left(\frac{2}{r}+\frac{F^{\prime \prime}(R)}{F^{\prime}(R)} \frac{d R}{d r}\right)-\frac{1}{r^{2}} \frac{F^{\prime \prime}(R)}{F^{\prime}(R)} \frac{d^{2} R}{d r^{2}}-\frac{1}{r^{2}} \frac{F^{\prime \prime \prime}(R)}{F^{\prime}(R)}\left(\frac{d R}{d r}\right)^{2}\right]=0 .
\end{gathered}
$$

Furthermore, as already mentioned, the variation with respect to $R$ leads again to Eq. (7). With this approach, Eq. (13) does not contain an explicit (nontrivial) dependence on $\alpha(r)$, while Eq. (14) does not contain an explicit dependence on $B(r)$. In 
order to look for exact solutions, the strategy is to make a suitable ansatz for $R=R(r)$ or to make an ansatz for $\alpha(r)$. In the next subsections, we review the examples of $\mathrm{BH}$ solutions we are interested in.

\section{A. Constant curvature case}

In the constant Ricci scalar case one has $R=R_{0}$. From Eq. (14) we immediately obtain

$$
\alpha(r)=\text { const. }
$$

Thus, if $F^{\prime}\left(R_{0}\right) \neq 0$, Eq. (13) leads to the topological Schwarzschild-AdS solution, ${ }^{3}$

$B(r)=k-\frac{c}{r}-\frac{\Lambda r^{2}}{3}, \quad \Lambda=\frac{R_{0} F^{\prime}\left(R_{0}\right)-F\left(R_{0}\right)}{2 F^{\prime}\left(R_{0}\right)}<0$,

where $c$ is a free integration constant. Finally, from Eq. (7) one has

$$
R_{0}=4 \Lambda,
$$

such that $\Lambda=F\left(R_{0}\right) /\left(2 F^{\prime}\left(R_{0}\right)\right)$.

\section{B. Solutions with $\alpha(r)=$ const}

Equation (14) with $\alpha(r)=$ const leads to [11,12],

$$
F^{\prime}(R)=a r+b,
$$

where $a, b$ are constant parameters. The form of $B(r)$ can be derived by taking the derivative with respect to $r$ of Eq. (13), but in general it is not possible to fully reconstruct the corresponding $F(R)$ model (see Refs. [12,13] for details). On the other hand, when $a=0$ we recover the constant Ricci scalar case already treated in the preceding subsection, while if one poses $b=0$ we get

$$
B(r)=\frac{k}{2}+\frac{c}{r^{2}}+\lambda r^{2},
$$

where $c, \lambda$ are integration constants. The Ricci scalar reads

$$
R=-12 \lambda+\frac{k}{r^{2}}
$$

and by using Eq. (18) one easily reconstructs the model as

$$
F(R)=2 a k \sqrt{k(R+12 \lambda)} .
$$

\footnotetext{
${ }^{3}$ Since in this paper we are interested in black hole solutions with a well-defined temperature we do not consider de Sitter metrics with two horizons.
}

Note that in this case only one free integration constant $c$ appears in the metric.

\section{Clifton-Barrow solutions}

Consider the Lagrangian

$$
F(R)=\frac{R^{\delta+1}}{\kappa}, \quad \delta \neq 1,
$$

with $\kappa$ being a dimensional parameter. One looks for solutions described by the SSS metrics with $\alpha(r) \neq 0$, namely

$$
\mathrm{e}^{2 \alpha(r)}=\left(\frac{r}{r_{0}}\right)^{2 a}
$$

where $a$ is a number and $r_{0}$ a dimensional constant. We also assume

$$
R=\frac{R_{0}}{r^{2}}
$$

In this case, Eqs. (13)-(14) are solved by ( $k=1$ in Ref. [8], $k$ generic in Ref. [20])

$$
\alpha(r)=\log \left[\left(\frac{r}{r_{0}}\right)^{a}\right], \quad B(r)=B_{0}\left(k-\frac{c}{r^{b}}\right),
$$

where $c$ is a free integration constant and $R_{0}, B_{0}, a, b$ are functions of the parameter $\delta$,

$$
\begin{aligned}
R_{0} & =\frac{6 \delta k(1+\delta)}{\left(2 \delta^{2}+2 \delta-1\right)}, \\
B_{0} & =\frac{(1-\delta)^{2}}{\left(1-2 \delta+4 \delta^{2}\right)\left(1-2 \delta-2 \delta^{2}\right)}, \\
a & =\frac{\delta(1+2 \delta)}{(1-\delta)} \\
b & =\frac{\left(1-2 \delta+4 \delta^{2}\right)}{(1-\delta)} .
\end{aligned}
$$

We also observe that the following relation holds true:

$$
b=a-2 \delta+1 .
$$

When $\delta=-1 / 2$ one has $\alpha(r)=$ const and we recover the model (21) with $\lambda=0$ and solution (19). The case $\delta=1$ has to be considered separately and corresponds to the scale invariant model $F(R) \sim R^{2}$ (see for example [42]) and is not investigated in this paper.

It is also possible to add to the Clifton-Barrow model in (22) a cosmological constant. An explicit example is the following: $a=2$; thus $\delta=-2$. The corresponding model with cosmological constant is given by [13] 


$$
F(R)=\frac{1}{\kappa}\left(\frac{1}{R}-\lambda\right)
$$

When $k \neq 0$, the model admits the topological SSS solution (6) with

$\mathrm{e}^{2 \alpha(r)}=\left(\frac{r}{r_{0}}\right)^{4}, \quad B(r)=-\frac{k}{7}+\frac{c}{r^{7}}+\frac{8 \lambda}{15 r^{2}}, \quad R=\frac{4 k}{r^{2}}$.

Furthermore, when $\lambda \neq 0$ the model in (28), after the redefinition $\lambda \rightarrow 6 k / \lambda^{2}$, leads to the solution [12]

$\mathrm{e}^{2 \alpha(r)}=\left(\frac{r}{r_{0}}\right), \quad B(r)=\frac{4}{7}\left(k+\frac{c}{r^{7 / 2}}-\frac{7 \lambda r}{36}\right), \quad R=\frac{\lambda}{r}$.

\section{FIRST LAW AND BH ENERGY IN $F(R)$ GRAVITY}

In this section, following Ref. [20], we propose a simple method to obtain the black hole energy in $F(R)$ gravity by starting from the first law of thermodynamics (see also Refs. [15-17]).

We recall that a SSS solution in the form of (6) describes a black hole with a single event horizon with radius $r=r_{H}$ when there exists a single $r_{H}>0$ such that

$$
B\left(r_{H}\right)=0, \quad 0<\left.\frac{d B(r)}{d r}\right|_{r=r_{H}} .
$$

In this way, $0<d B(r) /\left.d r\right|_{r_{H}}$ leads to a positive Killing surface gravity

$$
\kappa_{K}=\left.\mathrm{e}^{\alpha\left(r_{H}\right)} \frac{d B(r)}{d r}\right|_{r=r_{H}} .
$$

The metric signature $(-+++)$ is preserved for $r_{H}<r$, while it is violated when $r<r_{H}$. In other words, inside of the horizon, the coordinate $r$ plays the role of the time, $t$ plays the role of a spatial coordinate, and the metric becomes dynamic.

The metrics presented in the preceding section describe a black hole at least for some choices of the horizon topology. For example, it is well known that the Schwarzschild-AdS metric in (16) can describe a black hole with various topologies when $\Lambda<0$ (see Ref. [43]), but if $\Lambda=0$ we obtain a black hole only for $k=1$ and $0<c$.

Given a $\mathrm{BH}$ solution within a $F(R)$-modified gravity model, it is well known that the entropy and the related Hawking temperature can be computed by making use of independent approaches. In the case of the black hole entropy, the Wald method gives [44]

$$
S_{W}=(4 \pi) V_{k} r_{H}^{2} F^{\prime}\left(R_{H}\right),
$$

where the pedex $H$ denotes a quantity evaluated with respect to $r=r_{H}$. The Killing-Hawking temperature [45] can be derived, for instance, with the tunneling method $[46,47]$ and reads

$$
T_{K}=\frac{\kappa_{K}}{2 \pi} \equiv \frac{\mathrm{e}^{\alpha\left(r_{H}\right)}}{4 \pi} \frac{d B\left(r_{H}\right)}{d r} .
$$

Thus, from Eq. (13) evaluated on the BH horizon we may derive a first law of thermodynamics where the Killing temperature emerges in a natural way as follows:

$$
\begin{aligned}
T_{k} d S_{W}= & \mathrm{e}^{\alpha\left(r_{H}\right)} V_{k}\left(2 k F^{\prime}\left(R_{H}\right)-\left(R_{H} F^{\prime}\left(R_{H}\right)\right.\right. \\
& \left.\left.-F\left(R_{H}\right) r_{H}^{2}\right)\right) d r_{H} .
\end{aligned}
$$

Here, we have used the condition $B\left(r_{H}\right)=0$ and we have multiplied by $d r_{H}$. An important remark is in order. The relation

$d S_{W}=(4 \pi) V_{k}\left(2 r_{H} F^{\prime}\left(R_{H}\right) d r_{H}+\left.r_{H}^{2} F^{\prime \prime}\left(R_{H}\right)\left(\frac{d R}{d r}\right)\right|_{H} d r_{H}\right)$

is valid only if

$$
d R_{H}=\left.\left(\frac{d R}{d r}\right)\right|_{H} d r_{H}
$$

It means that the on-shell form of the Ricci scalar does not have to depend on the integration constant(s) of the solution. In this case the first law holds true,

$$
T_{K} d S_{W}=d E_{K},
$$

and leads to the identification

$d E_{K}=\mathrm{e}^{\alpha\left(r_{H}\right)} V_{k}\left(2 k F^{\prime}\left(R_{H}\right)-\left(R_{H} F^{\prime}\left(R_{H}\right)-F\left(R_{H}\right) r_{H}^{2}\right)\right) d r_{H}$.

Thus, at least in the case where only an integration constant appears in the black hole solution, we have an explicit expression for the $\mathrm{BH}$ energy in $F(R)$ gravity,

$E_{K}:=V_{k} \int \mathrm{e}^{\alpha\left(r_{H}\right)}\left(2 k F^{\prime}\left(R_{H}\right)-\left(R_{H} F^{\prime}\left(R_{H}\right)-F\left(R_{H}\right) r_{H}^{2}\right)\right) d r_{H}$.

The condition (36) looks restrictive, but it holds for a large class of static black holes in $F(R)$ gravity. For these solutions, the first law is a robust argument for the definition of the BH energy or mass. In particular, these considerations are valid for the $F(R)$ models presented in the previous section. 


\section{THE EVALUATION OF THE ACTION GROWTH IN $\boldsymbol{F}(\boldsymbol{R})$ GRAVITY}

In this section, we start recalling the approach described in Ref. [2] and used within $F(R)$ gravity in other papers (see for example Refs. [7,48]). In Ref. [4] this approach has been rigorously proved to give the correct answer. Within a holographic scenario, the CA conjecture tells us that one can compute the complexity growth by the evaluation of the action growth in the time, action defined with respect to the so-called WdW patch. For large time, one may consider only the bulk on-shell action associated with the black hole solution evaluated in the interior region of the black hole.

Motivated by these considerations, we compute the action growth in $F(R)$-modified gravity making use of our minisuperspace approach and working only with the bulk action (12). We are interested in computing the action growth associated with the interior of the black holes, namely for $r<r_{H}$, where the metric becomes dynamics. Thus, if we replace $r$ with a time coordinate and $t$ with a space coordinate,

$$
r=T, \quad t=\rho, \quad 0<T<r_{H},
$$

we can see the interior metric as a spherically symmetric dynamical space-time. The metric (6), after the redefinition $B(r) \rightarrow-B(T)$, can be rewritten as

$$
\begin{aligned}
d s^{2} & =-\frac{d T^{2}}{B(T)}+\mathrm{e}^{2 \alpha(T)} B(T) d \rho^{2}+T^{2} d \Omega_{k}^{2} \\
& =\gamma_{i j}\left(x^{i}\right) d x^{i} d x^{j}+(\mathcal{R})^{2} d \Omega_{k}^{2},
\end{aligned}
$$

where $\gamma_{i j}\left(x^{i}\right)$ is the reduced metric with respect to the coordinates $x^{i}=(T, \rho), \mathcal{R}\left(x^{i}\right)=T$ is the areal radius, and $B(T)$ is positive in the given range of $T$. In a dynamical case we lose the timelike Killing vector field and the Killing formalism becomes meaningless. On the other hand, one can use the covariant Hayward formalism [49]. The trapping (event horizon) is located at

$$
\chi=\gamma^{i j} \partial_{i} R\left(x^{i}\right) \partial_{j} R\left(x^{i}\right)=0,
$$

and one has $B\left(T_{H}\right)=0$. Furthermore, Hayward surface gravity is

$$
\kappa_{H}=\frac{1}{2} \square_{\gamma} \mathcal{R}\left(x^{i}\right)_{H},
$$

where the d'Alambertian is referred to as the reduced metric. In our case

$$
\kappa_{H}=-\left.\frac{1}{2} \frac{d B(T)}{d T}\right|_{T=T_{H}} .
$$

Here, the role of the timelike Killing vector is played by the Kodama vector [50],

$$
K^{i}=\frac{1}{\sqrt{-\gamma}} \varepsilon^{i j} \partial_{j} R\left(x^{i}\right),
$$

where $\gamma$ is the determinant of the reduced metric $\gamma_{i j}\left(x^{i}\right)$ and $\epsilon^{i j}$ is the two-dimensional antisymmetric Levi-Civita tensor. Thus, we get

$$
K^{\mu}=\left(0, \mathrm{e}^{-\alpha(T)}, 0,0\right) .
$$

The action growth can be defined in a covariant way by means of

$$
C=\lim _{T \rightarrow r_{H}} K^{\mu} \partial_{\mu} \hat{I}
$$

where $\hat{I}$ is the bulk action (12).

For our class of black hole solutions within the modified gravitational theories described by $F(R)$, one has

$$
C=V_{k} \mathrm{e}^{-\alpha\left(r_{H}\right)} \int_{0}^{r_{H}} d T L(T),
$$

where the bulk Lagrangian is given by

$$
\begin{aligned}
L(T)= & e^{\alpha(T)}\left\{T^{2}\left(F(R)-F^{\prime}(R) R\right)\right. \\
& +2 F^{\prime}(R)\left(k-T \frac{d B(T)}{d T}-B(T)-2 T B(T) \frac{d \alpha(T)}{d T}\right) \\
& \left.-F^{\prime \prime}(R) \frac{d R}{d T} T^{2}\left(\frac{d B(T)}{d T}+2 B(T) \frac{d \alpha(T)}{d T}+\frac{4 B(T)}{T}\right)\right\},
\end{aligned}
$$

and must be evaluated on shell, $R=R(T)$ being a function of $T$,

$$
\begin{aligned}
R= & 3\left[\frac{d}{d T} B(T)\right] \frac{d}{d T} \alpha(T)+2 B(T)\left[\frac{d}{d T} \alpha(T)\right]^{2} \\
& +\frac{d^{2}}{d T^{2}} B(T)+2 B(T) \frac{d^{2}}{d T^{2}} \alpha(T) \\
& +4 \frac{\frac{d}{d T} B(T)}{T}+4 \frac{B(T) \frac{d}{d T} \alpha(T)}{T}+2 \frac{B(T)}{T^{2}}+\frac{2 k}{T^{2}} .
\end{aligned}
$$

\section{A. Action growth: $\alpha=0$ cases}

In this subsection, we calculate the action growth of the $F(R)$-black holes with metric (6) and $\alpha(r)=$ const. Without loss of generality we can pose $\alpha(r)=0$. Let us start with the constant Ricci curvature case $R=R_{0}$ analyzed in Sec. II A. Evaluating the Lagrangian (49) on the solution,

$B(T)=-k+\frac{c}{T}+\frac{\Lambda T^{2}}{3}, \quad \Lambda=\frac{R_{0} F^{\prime}\left(R_{0}\right)-F\left(R_{0}\right)}{2 F^{\prime}\left(R_{0}\right)}$,

one has 


$$
L(T)=4 F^{\prime}\left(R_{0}\right)\left(k-\Lambda T^{2}\right) .
$$

As a result we obtain for the action growth (48),

$$
C=4 V_{k} F^{\prime}(R)\left(k r_{H}-\frac{1}{3} \Lambda r_{H}^{3}\right) .
$$

By using the horizon condition $B\left(T_{H}\right)=0$, we get

$$
C=4 V_{k} F^{\prime}(R) c .
$$

In general, from (39), we can now identify

$$
E_{K}=2 V_{k} F^{\prime}\left(R_{H}\right) c .
$$

As a result one gets

$$
C=2 E_{K}
$$

This result is in agreement with the action growth computed by other method in Refs. [5,6,48]. One remark is in order. The expression (55) has been obtained by fixing the cosmological constant $\Lambda$ and the procedure is always valid when $\Lambda$ explicitly appears in the form of the $F(R)$ model [for example, $F(R) \propto R-2 \Lambda$ ]. However, when the cosmological constant is a second integration constant of the solution, an additional thermodynamical potential may contribute to the energy. It is the case, for instance, of $R^{2}$ gravity, where the scale invariance of the theory brings to the emergence of the length scale from the solution.

Let us come back to the model (21) discussed in Sec. II B, for which one has $\alpha(T)=0$, but nontrivial Ricci curvature. We have that the model admits the following interior $\mathrm{BH}$ solution,

$$
B(T)=-\frac{k}{2}-\frac{c}{T^{2}}-\lambda T^{2},
$$

with nonconstant Ricci scalar,

$$
R=-12 \lambda+\frac{k}{T^{2}}
$$

The on-shell Lagrangian is

$$
L(T)=6 a T\left(k+4 \lambda T^{2}\right) .
$$

Thus, the action growth results in

$$
C=6 V_{k} a\left(\frac{k r_{H}^{2}}{2}+\lambda r_{H}^{4}\right) \equiv-6 V_{K} a c .
$$

From (39) we have that the energy of the black hole under investigation is

$$
E_{K}=-3 V_{K} a c,
$$

and one obtains again the relation (56). This is a new result, similar to the case with constant Ricci scalar discussed above.

\section{B. Action growth: Clifton-Barrow models}

As an example of the nonconstant Ricci scalar case with $\alpha(r) \neq 0$, we compute the action growth for the CliftonBarrow models (22) discussed in Sec. II C. The interior BH solution reads

$\alpha(T)=\log \left[\left(\frac{T}{r_{0}}\right)^{a}\right], \quad B(T)=B_{0}\left(-k+\frac{c}{T^{b}}\right), \quad R=\frac{R_{0}}{T^{2}}$

where $R_{0}, B_{0}, a$ and $b$ are given by (26). By using the definitions in (48)-(49) and the condition (27), the action growth results in

$C=\frac{V_{k} e^{-\alpha(T)}}{\kappa r_{0}^{a}} R_{0}^{\delta} B_{0}\left(1-\delta^{2}\right) k r_{H}^{b} \equiv \frac{V_{k} e^{-\alpha(T)}}{\kappa r_{0}^{a}} R_{0}^{\delta} B_{0}\left(1-\delta^{2}\right) c$.

On the other hand, the Killing BH energy for a CliftonBarrow $\mathrm{BH}$ is derived as

$$
E_{K}=2\left(1-\delta^{2}\right) \frac{V_{k}}{r_{0}^{a} \kappa} R_{0}^{\delta} B_{0} c
$$

and, as a consequence, one has

$$
C=2 \mathrm{e}^{-\alpha\left(r_{H}\right)} E_{K}
$$

We return later to this result.

As a further example, we consider now the model (28) with interior BH solution,

$\mathrm{e}^{2 \alpha(T)}=\left(\frac{T}{r_{0}}\right)^{4}, \quad B(T)=\frac{k}{7}-\frac{c}{T^{7}}-\frac{8 \lambda}{15 T^{2}}, \quad R=\frac{4 k}{T^{2}}$,

where we recall that $k \neq 0$. For the action growth one obtains

$$
C=\frac{3 V_{k} \mathrm{e}^{-\alpha\left(r_{H}\right)}}{4 r_{0}^{2} \kappa}\left(\frac{k r_{H}^{7}}{7}-\frac{8 \lambda r_{H}^{5}}{15}\right) \equiv \frac{3 \mathrm{e}^{-\alpha\left(r_{H}\right)} c}{4 r_{0}^{2} \kappa} .
$$

Since the BH Killing energy computed with the static external metric reads

$$
E_{K}=\frac{3 c}{8 r_{0}^{2} \kappa},
$$

we see that the relation (65) holds true again. The result is confirmed even in the case of the interior BH solution 


$$
\begin{aligned}
\mathrm{e}^{2 \alpha(T)} & =\left(\frac{T}{r_{0}}\right), \\
B(T) & =-\frac{4}{7}\left(k+\frac{c}{T^{7 / 2}}-\frac{7 \lambda T}{36}\right), \\
R & =\frac{\lambda}{T},
\end{aligned}
$$

which can still be inferred from the model (28) after the redefinition $\lambda \rightarrow 6 k / \lambda^{2}$. Now the action growth is

$C=\frac{8 V_{k} \mathrm{e}^{-\alpha\left(r_{H}\right)}}{63 \lambda^{2} \kappa\left(r_{0}\right)^{1 / 2}} r_{H}^{7 / 2}\left(-36 k+7 \lambda r_{H}\right) \equiv \frac{32 V_{K} \mathrm{e}^{-\alpha\left(r_{H}\right)} c}{7 \kappa \lambda^{2}\left(r_{0}\right)^{1 / 2}}$,

while the Killing energy of the black hole is derived as

$$
E_{K}=\frac{16 V_{K} c}{7 \kappa \lambda^{2}\left(r_{0}\right)^{1 / 2}}
$$

It follows that for these classes of black hole solutions with $\alpha(r) \neq 0$, the action growth has the universal form (65); namely the Kodama-Hayward energy,

$$
E_{H}=\mathrm{e}^{-\alpha\left(r_{H}\right)} E_{K}
$$

appears. When $\alpha=0$ we recover the relation (56).

\section{DESER-SARIOGLU-TEKIN BLACK HOLES}

So far, we have investigated in detail the $\mathrm{BH}$ solution within $F(R)$-modified gravity, and we have obtained a quite general result expressed by (65). In this section we present another specific example of modified gravity for which we can make use of the minisuperspace approach.

In Ref. [51], Deser et al. presented an interesting model based on a Weyl correction to GR for which they provide an exact SSS BH solution. The model including the cosmological constant has the following action,

$$
I=\frac{1}{2 \kappa^{2}} \int_{\mathcal{M}} d^{4} x \sqrt{-g}(R-2 \Lambda+\sqrt{3} \sigma \sqrt{W})
$$

where $\Lambda$ is the cosmological constant, $\sigma$ is a real dimensionless parameter and $W=C_{\mu \nu \xi \sigma} C^{\mu \nu \xi \sigma}$ is the square of the Weyl tensor,

$$
W=\frac{1}{3} R^{2}-2 R_{\mu \nu} R^{\mu \nu}+R_{\mu \nu \xi \sigma} R^{\mu \nu \xi \sigma},
$$

$R_{\mu \nu}$ and $R_{\mu \nu \sigma \xi}$ being the Ricci and the Riemann tensors, respectively. For $\sigma=0$ the Weyl contribution turns off and the action of the $\Lambda \mathrm{CDM}$ model is recovered for $\kappa^{2}=16 \pi G_{N}$, with $G_{N}$ being the Newton constant. A key point is the following: for the SSS metric (6) the square of the Weyl tensor is a perfect square and reads

$$
\begin{aligned}
W= & \frac{1}{3}\left[\frac{1}{r^{2}}\left[r^{2}\left(\frac{d^{2} B(r)}{d r^{2}}\right)+2(B(r)-k)-2 r\left(\frac{d B(r)}{d r}\right)\right]\right. \\
& \left.+\frac{1}{r}\left[3 r\left(\frac{d B(r)}{d r}\right)\left(\frac{d \alpha(r)}{d r}\right)-2 B(r)\left(\frac{d \alpha(r)}{d r}-r\left(\frac{d^{2} \alpha(r)}{d r^{2}}+\left(\frac{d \alpha(r)}{d r}\right)^{2}\right)\right)\right]\right]^{2} .
\end{aligned}
$$

After integration by parts, we are able to separate the action of the bulk from the boundary terms (see Appendix C) as $I=\frac{V_{k}}{2 \kappa^{2}} \int d t \int d r \mathrm{e}^{\alpha(r)}\left(-2 \Lambda r^{2}+2 k(1-\epsilon \sigma)+2 B(r)(1-\epsilon \sigma)+2 r \frac{d B(r)}{d r}(1-4 \epsilon \sigma)+2 r B(r) \frac{d \alpha(r)}{d r}(2-5 \epsilon \sigma)\right)+B T$,

where

$$
\begin{aligned}
B T & =-\frac{V_{k}}{2 \kappa^{2}} \int d t \int d r \frac{d}{d r}\left[\mathrm{e}^{\alpha(r)} r^{2}\left(\frac{d B(r)}{d r}+2 B(r) \frac{d \alpha(r)}{d r}+\frac{4 B(r)}{r}\right)(1-\epsilon \sigma)\right] \\
& =-\frac{V_{k} \Delta t}{2 \kappa^{2}}\left[\mathrm{e}^{\alpha(r)} r^{2}\left(\frac{d B(r)}{d r}+2 B(r) \frac{d \alpha(r)}{d r}+\frac{4 B(r)}{r}\right)(1-\epsilon \sigma)\right] .
\end{aligned}
$$

In this expression, the parameter $\epsilon= \pm 1$ must be set in order to have $\sqrt{W}=|\sqrt{W}|$. The bulk action is obtained by subtracting the boundary term, namely

$$
\hat{I}=\frac{V_{k}}{2 \kappa^{2}} \int d t \int d r \mathrm{e}^{\alpha(r)}\left(-2 \Lambda r^{2}+2 k(1-\epsilon \sigma)+2 B(r)(1-\epsilon \sigma)+2 r \frac{d B(r)}{d r}(1-4 \epsilon \sigma)+2 r B(r) \frac{d \alpha(r)}{d r}(2-5 \epsilon \sigma)\right) .
$$


The field equations are derived by making the variation of the bulk action with respect to $\alpha(r)$ and $B(r)$ and read

$$
\begin{aligned}
& \frac{V_{k}}{2 \kappa} \mathrm{e}^{\alpha(r)}\left[(1-\epsilon \sigma)\left(k-B(r)-r \frac{d B(r)}{d r}\right)\right. \\
& \left.+3 \epsilon \sigma B(r)-\Lambda r^{2}\right]=0, \\
& \frac{V_{k}}{2 \kappa} \mathrm{e}^{\alpha(r)}\left[3 \epsilon \sigma+\frac{d \alpha(r)}{d r}(1-\epsilon \sigma) r\right]=0 .
\end{aligned}
$$

Note that for the SSS metric the field equations of this theory are at the second order. The general solution is given by $[20,51]$

$$
\begin{aligned}
\alpha(r) & =\log \left[\frac{r}{r_{0}}\right]^{\frac{3 \epsilon \sigma}{\epsilon \sigma-1}}, \\
B(r) & =k \frac{(1-\epsilon \sigma)}{(1-4 \epsilon \sigma)}-c r^{-\frac{1-4 \epsilon \sigma}{1-\epsilon \sigma}}-\Lambda \frac{r^{2}}{3(1-2 \epsilon \sigma)}, \\
\sigma & \neq \pm 1, \pm \frac{1}{4},
\end{aligned}
$$

where $r_{0}$ has been introduced for dimensional reasons, and $c$ is an integration constant. This solution describes a black hole with event horizon located at $B\left(r_{H}\right)=0$. Thus, if one uses the Killing temperature and the Wald entropy [52],

$$
\begin{aligned}
T_{K} & =\frac{1}{4 \pi}\left(\frac{r_{H}}{r_{0}}\right)^{\frac{3 \epsilon \epsilon}{\epsilon \sigma-1}}\left(c\left(\frac{1-4 \epsilon \sigma}{1-\epsilon \sigma}\right) r_{H}^{\frac{-3 \epsilon \sigma}{1-\epsilon \sigma}}-2 \Lambda \frac{r_{H}}{3(1-2 \epsilon \sigma)}\right), \\
S_{W} & =(4 \pi) \frac{V_{k} r_{H}^{2}}{2 \kappa}(1-\epsilon \sigma),
\end{aligned}
$$

it is easy to see that Eq. (79) evaluated on the horizon leads to the first law of thermodynamics, namely

$$
T_{K} d S_{W}=\frac{V_{k} \mathrm{e}^{\alpha\left(r_{H}\right)}}{\kappa}\left[(1-\epsilon \sigma) k-\Lambda r_{H}^{2}\right] \equiv d E_{K} .
$$

Thus, we can identify the BH energy as

$E_{K}:=\frac{V_{k}}{\kappa} \int \mathrm{e}^{\alpha\left(r_{H}\right)}\left[(1-\epsilon \sigma) k-\Lambda r_{H}^{2}\right] d r_{H}=\frac{V_{k} c}{\kappa r_{0}^{\frac{3 \epsilon \sigma}{\epsilon \sigma}}}(1-\epsilon \sigma)$,

where we have taken into account that on the horizon $B\left(r_{H}\right)=0$.

The growth action can be computed in an analogue way of the $F(R)$ case by starting from (78) and the result is

$$
C=\mathrm{e}^{-\alpha\left(r_{H}\right)} E_{K} \frac{(2-5 \epsilon \sigma)}{(1-\epsilon \sigma)} \leq 2 \mathrm{e}^{-\alpha\left(r_{H}\right)} E_{K} .
$$

In this case of the modified gravity model, the action growth does not coincide with the double of the Kodama energy, but is still proportional to it and, more importantly, bounded by twice its value as long as $0<\epsilon \sigma$, which is in accord with the general complexity bound as usually stated. In the contrary case $\epsilon \sigma<0$ and the bound is violated. As a check, when $\sigma$ goes to 0 , one gets the result of general relativity.

\section{A BIT OF BLACK HOLE PHENOMENOLOGY}

What we say in this section is strictly valid in Einstein's theory of gravity and then argued to hold for more general models. Black holes radiate away their mass in a certain lifetime. The efficiency of particle emission from black holes is clearly an important issue of the evaporation phenomenon. Beyond this, it is also relevant to interpret the result on the action grow that we obtained in some models of modified gravity. In particular, we justify the use of nonrotating uncharged solutions.

As is well known, the temperature of a black hole in GR is inversely proportional to its total mass, $M$, which includes the gravitational contribution, and the horizon area $A$ is proportional to $M^{2}$, so the total power emitted $P$ is proportional to $A T^{4}$, or $M^{-2}$. From this it follows that the lifetime $t_{l}=M / P$ is proportional to $M^{3}$. To state a number, black holes formed by stellar collapse having $M_{\odot} \leq M$, where $M_{\odot}$ is the solar mass, have a lifetime of order $10^{66} \mathrm{yr}$. Therefore the thermal emission is physically insignificant for such black holes, although still very important theoretically. However, it is relevant for primordial black holes, for which $M$ could be less than $10^{15} \mathrm{~g}$, and the corresponding lifetime less than the age of the Universe.

The problem is how rapidly a charged rotating black hole discharges and spins down. The main difference in the rapidity of the two processes can be seen as follows. Given the $\mathrm{BH}$ angular momentum $J$ and the $\mathrm{BH}$ charge $Q$, the two parameters

$$
a_{*}=\frac{J}{M^{2}}, \quad Q_{*}=\frac{Q}{M},
$$

are constrained by the inequality

$$
a_{*}^{2}+Q_{*}^{2} \leq 1 .
$$

This is because the solutions with $1<a_{*}^{2}+Q_{*}^{2}$ do not describe black holes but exhibit, as a rule, one or more naked singularities. A charged emitted particle with mass $m$ carries off $n$ units of the fundamental charge, say $\Delta Q=n e$, and an angular momentum $-\Delta J=m$, both of order unity. Hence due to the constraint the number of charged particles needed to neutralize the hole is $Q /(n e)$, which is at most of order $M$, and the number of particles needed to spin down the hole is $J / m$, which is at most of order $M^{2}$. Thus the hole can discharge quickly [53-55] but the loss of angular momentum requires the same number of particles as the loss of mass. The question of the evolution of a rotating black hole was analyzed by Page in Refs. $[56,57]$ in great detail. By considering all the known particles with masses less than $20 \mathrm{Mev}$, the temperature of a black hole with mass 
of order $10^{16}$ grams, he found that the emission of angular momentum increases greatly with $a_{*}$. Moreover, more than one half of the energy is emitted after $a_{*}$ reaches a small value of the order of 0.06 . From this point the power is within $1 \%$ of its Schwarzschild value and therefore the earlier assumption that decaying black holes have negligible rotation is valid.

These properties are challenged by the black holes of modified gravity, although the main argument should retain its strength, because the standard model Lagrangian, describing the matter part of the system, is not modified in the present considerations and the only additional particle in the gravitational sector (other than the massless graviton) is a massive scalar. In Page's time one did not consider the emission of dark matter particles, and we too avoid this question here.

But it is clear that the black hole will emit several species of massless and massive particles depending on its temperature. In this case the total luminosity of the black hole can be computed by summing over all particle species. Don Page was able to estimate the total power emitted: taking into account four kinds of neutrinos $\left(\nu_{e}, \nu_{\mu}\right.$ and the two antineutrinos), the photon and the graviton, for $10^{17} \mathrm{~g}<M$ his result was

$$
P=2.28 \times 10^{-54} L_{\odot}\left(M_{\odot} / M\right)^{2},
$$

where $81.4 \%$ is in the four kinds of neutrinos, $16.7 \%$ is in photons and $1.9 \%$ is in gravitons. Here, $M_{\odot}=1.99 \times$ $10^{33} \mathrm{~g}$ is the solar mass and $L_{\odot}=3.9 \times 10^{33} \mathrm{erg} \mathrm{sec}^{-1}$ is the solar luminosity. For $5 \times 10^{14}<M<10^{17}$ the black hole emits ultrarelativistic $e^{ \pm}$which may be treated as massless fermions, and the power is

$$
P=4.07 \times 10^{-54} L_{\odot}\left(M_{\odot} / M\right)^{2},
$$

of which $45 \%$ is in electrons and positrons, $45 \%$ is in neutrinos, $9 \%$ is in photons and only $1 \%$ in gravitons. In all, most of the energy is radiated in the form of massless or nearly massless particles, as was to be expected on general grounds for a low temperature object. Moreover, the bulk of the radiation appears in standard model particles, rather than gravitons.

The black holes of modified gravity we are considering presently have a finite temperature and entropy and obey the first law of thermodynamics for a suitable defined thermodynamics energy. Thus they radiate away their energy via Hawking steady emission for most of their lifetimes. They do this by emitting particles of the standard model, which is left untouched in modified gravity. Moreover, no new gravitational excitation is introduced except for a massive scalar, so we may still consider the evaporation rate of black holes in modified gravity, for not too small masses, as substantially identical as for asymptotically flat black holes in general relativity. ${ }^{4}$ Of course filling in the details (like the precise percentages for example) deserves a more careful study.

The conclusions we can draw from the above discussion and the examples we gave for the explicit solutions is that the simple scaling of the action grow with the thermodynamics energy is a direct consequence of the universality of Hawking radiation for a given particle spectrum. In particular, it is largely independent on the gravitational sector and massive states are not radiated anyway. So one expects the same complexity as in GR, if gravity is treated classically.

One can see the connection with Hawking radiation quantitatively. In GR the Hamiltonian on a three-surface $\Sigma$ bounded by a sphere which is part of a horizon has the form

$$
\begin{aligned}
\mathcal{H}= & \text { bulk term }-\frac{1}{8 \pi G_{N}} \int_{\mathcal{M}}\left(\kappa-16 \pi h^{-1 / 2} P^{i j} N_{i} \xi_{j}\right) d A \\
& + \text { terms at infinity, }
\end{aligned}
$$

where $\kappa$ is the surface gravity, $N_{i}$ the shift, and $\xi_{j}$ the normal to $\mathcal{M}$ within the three-surface and where $h$ is the determinant of the induced metric on the three-surface $\Sigma$. On shell the bulk term vanishes because it is a constraint, the momentum term also vanishes in a static geometry or when the shift is taken to vanish on the horizon, while the term at infinity is absent if $\Sigma$ is internal to the horizon. Identifying the temperature $T=$ $\kappa / 2 \pi$ and the entropy $S_{\mathrm{BH}}=A / 4 G_{N}$ as usual, the action grow bound is $\dot{I}=T S_{\mathrm{BH}} \leq 2 E$, or by taking derivatives ${ }^{5}$

$$
\dot{S}_{\mathrm{BH}} \leq-2 P / T,
$$

where $P$ is the power emitted by the $\mathrm{BH}$. We have to consider now the entropy carried away by the Hawking radiation, say $\dot{S}_{\text {rad }}$. By the generalized second law,

$$
0 \leq \dot{S}_{\mathrm{BH}}+\dot{S}_{\mathrm{rad}}
$$

so finally

$$
2 P / T \leq \dot{S}_{\text {rad }}
$$

We should note that one usually expects $P / T \leq \dot{S}_{\text {rad }}$ by conventional thermodynamics.

If one accepts Pendry's [21] universal bound on the entropy flow out of a thermal source radiating in vacuum (like the black hole)

\footnotetext{
${ }^{4}$ At least if we treat gravity (modified or not) as a classical external field.

${ }^{5}$ The time derivative of an inequality does not necessarily respect the inequality, but if the entropy increases with energy that is the case. The only exception would be systems with negative temperature.
} 


$$
\dot{S}_{\text {rad }} \leq\left(\frac{\pi P}{3}\right)^{1 / 2}
$$

(we remember that $k_{B}=1$ in our units) then one gets for the power the limit

$$
P \leq \frac{\pi T^{2}}{12}
$$

As a qualification, the Pendry inequality holds only for outward flow of energy and therefore it does not represent the maximum rate of cooling of the black hole. This is easily disposed of: since we took $P=-\dot{E}$, the left-hand side of Eq. (95) should be replaced by $\sum_{s} \bar{\Gamma}_{s} P_{s}$, where $\bar{\Gamma}_{s}$ is the average over energy of the transmission coefficient of the potential barrier surrounding the black hole for a particle species $s$. It is a number of order 1 . This is because the fraction $1-\sum_{s} \bar{\Gamma}_{s} P_{s}$ of the power is reflected back into the hole. The left-hand side is just the total power radiated via Hawking radiation by a Schwarzschild black hole, which saturates the inequality, and was used by Bekenstein [23] long ago to infer the one-dimensional character of a black hole considered as an information transmission channel. In fact, the inequality (94) can be easily violated by transmitting over many parallel channels. The result for the action grow in the modified gravity models considered here indicates the validity of the same bound for the power emitted, provided the power in these equations measures the rate of emission of the thermodynamical energy as defined in the text.

For different gravitational actions the particle spectrum sometimes changes radically. For example, adding a term $\sim R_{\mu \nu} R^{\mu \nu}$ introduces a massive spin two ghost, but what is the complexity of a negative norm state? However in one case the Weyl correction discussed above was in agreement with the standard complexity bound $(0<\epsilon \sigma)$; in another it was not $(\epsilon \sigma<0)$. It would be interesting to test whether in this case the theory contains ghostlike excitations, which would violate the action grow bound.

\section{CONCLUSIONS}

In this paper we have investigated the general form of the action growth for some modified gravity black hole solutions. Within this more general framework different to the one of GR, new vacuum black hole solutions with nonvanishing curvature may be found. In our analysis, we have considered several $\mathrm{BH}$ solutions where only one integration constant is present. Thus, by making use of the first law of BH thermodynamics in these modified gravity models, we have shown that the energy of our black holes is always proportional to the integration constant associated with the solution. We should note that within the class of modified gravity models we are interested in, the first law can be derived from the equations of motion and making use of the Killing temperature and the Wald entropy and this fact seems to be a robust argument to substantiate the definition of the BH energy we have made use of. In the case of solutions with constant Ricci curvature, we have confirmed the results of Refs. [5,6]; namely the action growth corresponds to the double of the Killing energy, in agreement with the result of Brown et al. in general relativity [2]. On the other hand, for solutions with nonconstant Ricci curvature, the Kodama-Hayward $\mathrm{BH}$ energy emerges in the action growth. We recall that the Kodama-Hayward energy is different to the Killing one due to the different expression of the Kodama and Killing vectors associated with dirty $\mathrm{BHs}$, and they coincide only when $g_{00}(r) g_{11}(r)=-1$. Our result is not surprising since the Hayward formalism is covariant and valid for spherically symmetric dynamical space-times.

In the last part of our work, we considered a modified gravity model based on a Weyl correction of gravity with an exact $\mathrm{BH}$ solution and we have derived the form of the related action growth, which is still proportional to the Kodama-Hayward energy of the black hole itself. In one case the GR bound was satisfied; in another it was not. We argue that the theory could contain a ghostlike excitation.

To interpret physically the obtained result, we argued on the basis of some black hole phenomenology that the complexity bound as expressed by the action grow is tightly related to the Hawking radiation process. Since the particle spectrum of $F(R)$ gravity is just the same as for GR, apart from a massive scalar, and the standard matter Lagrangian describing the matter sector is left untouched, the black holes of modified gravity radiate away their mass in essentially the same way as in GR. In fact, writing the action in the Einstein frame, a scalar degree of freedom appears which is really a masked metrical invariant of the Jordan frame. This formulation of the theory has been studied elsewhere in the cited references, but for constant scalar field. Our description can be interpreted in the Einstein frame as the presence of a nonconstant scalar field.

If the mass is defined as described in the text to represent the thermodynamical energy of the black holes, then the action grow must scale with this energy, as was actually found, and thus be the same as in GR up to numerical coefficients. This physical interpretation is not precise, since no detailed calculations were provided to fill in the details of the radiation process for general $f(R)$ models, except for the Brans-Dicke theory which has exactly the same black holes as general relativity, as was shown by Hawking long ago.

\section{APPENDIX A: BOUNDARY TERMS FOR SPACELIKE SURFACES}

In this appendix, we review some elementary aspects of induced geometry associated with an $r$-constant surface. Let us start by recalling the four-dimensional metric, 


$$
\begin{aligned}
d s^{2} & =g_{\mu \nu} d x^{\mu} d x^{\nu} \\
& =-\mathrm{e}^{2 \alpha(r)} B(r) d t^{2}+\frac{d r^{2}}{B(r)}+r^{2} s_{a b}\left(x^{a}\right) d x^{a} d x^{b},
\end{aligned}
$$

where $s_{a b}\left(x^{a}\right)$ is a two-dimensional "horizon metric." Let us denote by $n^{\mu}$ the unit normal vector to the surface $r$ constant, which reads

$$
n^{\mu}=\left(0, \frac{1}{\sqrt{g_{r r}}}, 0,0\right)=(0, \sqrt{B(r)}, 0,0) .
$$

The induced metric $h_{\alpha \beta}\left(x^{i}\right)$ of a surface with constant $r$ is given by

$$
h_{\alpha \beta}\left(x^{i}\right)=g_{\alpha \beta}\left(x^{\mu}\right)-n_{\alpha} n_{\beta},
$$

namely

$$
d h^{2}=-\mathrm{e}^{2 \alpha(r)} B(r) d t^{2}+r^{2} s_{a b} d x^{a} d x^{b},
$$

and this may represent a timelike, spacelike or null-like surface. One has $\sqrt{-h}=r^{2} e^{\alpha} \sqrt{B} \sqrt{s}$, and the related extrinsic curvature is defined as

$$
K=\nabla_{\alpha} n^{\alpha}=h^{\alpha \beta}\left(x^{i}\right) \nabla_{\beta} n_{\alpha} .
$$

Thus, one obtains

$$
K=\frac{\sqrt{B(r)}}{2}\left(\frac{1}{B(r)} \frac{d B(r)}{d r}+2 \frac{d \alpha(r)}{d r}+\frac{4}{r}\right),
$$

with scalar density

$\sqrt{-h} K=\sqrt{s} \frac{r^{2} \mathrm{e}^{\alpha(r)}}{2}\left(\frac{d B(r)}{d r}+2 \frac{d \alpha(r)}{d r} B(r)+\frac{4 B(r)}{r}\right)$.

In our work, $\sqrt{s}=V_{k}$. A direct computation of the boundary term in (4) leads to

$$
\begin{aligned}
B T & =-2 \int_{\partial \mathcal{M}} d^{3} x \sqrt{-h} F^{\prime}(R) K \\
& =-V_{k} \Delta t\left[F^{\prime}(R) \mathrm{e}^{\alpha(r)} r^{2}\left(\frac{d B(r)}{d r}+2 B(r) \frac{d \alpha(r)}{d r}+\frac{4 B(r)}{r}\right)\right],
\end{aligned}
$$

and we recover Eq. (8).

\section{APPENDIX B: CONSISTENCY OF THE ANZATZ WITH THE EQUATIONS OF MOTION}

In this appendix, we explicitly show that the equations of motion (13)-(14) obtained by inserting the metric ansatz (6) in the gravitational action of $F(R)$ gravity are equivalent to the $(0,0)$ and $(1,1)$ components of the general field equations (5) of the theory [in the vacuum case, the other nonzero components, namely the $(2,2)$ and $(3,3)$ components, are derived from the first two].

Let us rewrite Eq. (5) as

$$
\begin{aligned}
& F^{\prime}(R)\left(R_{\mu \nu}-\frac{1}{2} R g_{\mu \nu}\right)+\frac{1}{2} g_{\mu \nu}\left(R F^{\prime}(R)-F(R)\right) \\
& -\left(\nabla_{\mu} \nabla_{\nu}-g_{\mu \nu} \nabla_{\alpha} \nabla^{\alpha}\right) F^{\prime}(R)=0 .
\end{aligned}
$$

The $(0,0)$ and $(1,1)$ components of this equation with the metric (6) read

$$
\begin{aligned}
- & \left(\frac{B(r) \mathrm{e}^{2 \alpha(r)}}{2 r^{2}}\right)\left[r^{2}\left(R F^{\prime}(R)-F(R)\right)\right. \\
& -2 F^{\prime}(R)\left(k-B(r)-r \frac{d B(r)}{d r}\right) \\
& +2 B(r) F^{\prime \prime}(R) r^{2}\left[\frac{d^{2} R}{d r^{2}}+\left(\frac{2}{r}+\frac{d B(r) / d r}{2 B(r)}\right) \frac{d R}{d r}\right. \\
& \left.\left.+\frac{F^{\prime \prime \prime}(R)}{F^{\prime \prime}(R)}\left(\frac{d R}{d r}\right)^{2}\right]\right]=0, \\
( & \left.\frac{1}{2 B(r) r^{2}}\right)\left[r^{2}\left(R F^{\prime}(R)-F(R)\right)\right. \\
& -2 F^{\prime}(R)\left(k-B(r)-r \frac{d B(r)}{d r}\right) \\
& +4 F^{\prime}(R) r B(r) \frac{d \alpha(r)}{d r} \\
& \left.+F^{\prime \prime}(R) \frac{d R}{d r}\left(2 B(r) r^{2} \frac{d \alpha(r)}{d r}+4 B(r) r\right)\right]=0 .
\end{aligned}
$$

Thus, Eq. (B2) is equivalent to Eq. (13), while in order to obtain Eq. (14) we must substitute Eq. (B2) in Eq. (B3).

\section{APPENDIX C: THE BOUNDARY TERM OF THE WEYL MODEL}

In this appendix, following Ref. [36], we compute the boundary term for the Weyl model in (73) in the case of a SSS space-time. First of all, we recall the general form of the boundary term for such a kind of theory in the form of a surface integral with $r$ constant, namely

$$
B T=-2 \int_{\partial \mathcal{M}} d^{3} x \sqrt{-h} \Psi K,
$$

where we are using the parametrizations and the definitions in (A1)-(A7). Moreover, $\Psi$ is the trace of the tensor $\Psi^{i j}$,

$$
\Psi^{i j}=-2 h^{i \kappa} h^{j l} n^{\mu} n^{\nu} \phi_{\kappa \mu l \nu}, \quad \phi^{\kappa \mu l \nu}=\frac{d \mathcal{L}}{d R_{\kappa \nu l \nu}},
$$

where in our case 


$$
\mathcal{L}=\frac{1}{2 \kappa^{2}}(R-2 \Lambda+\sqrt{3} \sigma \sqrt{W})
$$

One has

$$
\frac{\delta \mathcal{L}}{\delta R_{\mu \nu \xi \sigma}}=\frac{1}{2 \kappa^{2}}\left\{\frac{1}{2}\left(g^{\mu \xi} g^{\nu \sigma}-g^{\mu \sigma} g^{\nu \xi}\right)+\frac{\sqrt{3} \sigma}{2 \sqrt{W}}\left[2 R^{\mu \nu \xi \sigma}-\left(g^{\mu \xi} R^{\nu \sigma}+g^{\nu \sigma} R^{\mu \xi}-g^{\mu \sigma} R^{\nu \xi}-g^{\nu \xi} R^{\mu \sigma}\right)+\frac{1}{3}\left(g^{\mu \xi} g^{\nu \sigma}-g^{\mu \sigma} g^{\nu \xi}\right) R\right]\right\} .
$$

By taking into account (A2) and the symmetries of the metric, it is easy to see that

$$
\Psi \equiv h_{i j} \Psi^{i j}=-2 h_{00} h^{00} h^{00} n^{r} n^{r} h_{00} h_{11} h_{00} h_{11} \phi^{0101}=2 \mathrm{e}^{2 \alpha(r)} \frac{d \mathcal{L}}{d R_{0101}} .
$$

A direct computation leads to

$$
\left(\frac{\delta \mathcal{L}}{\delta R_{0101}}\right)=\frac{1}{4 \kappa^{2}}\left[g^{00} g^{11}+\frac{\sqrt{3} \sigma}{\sqrt{C^{2}}}\left(2 R^{0101}-g^{00} R^{11}-g^{11} R^{00}+\frac{1}{3} g^{00} g^{11} R\right)\right]=\frac{1}{4 \kappa^{2} \mathrm{e}^{2 \alpha(r)}}(1-\epsilon \sigma) .
$$

We finally obtain

$$
\Psi=\frac{1}{2 \kappa^{2}}(1-\epsilon \sigma),
$$

and from Eq. (C1) with (A7) one has the result

$$
B T=-2 \int_{\partial \mathcal{M}} d^{3} x \sqrt{-h}\left(\frac{1-\epsilon \sigma}{2 \kappa^{2}}\right) K=-\frac{V_{k} \Delta t}{2 \kappa^{2}}(1-\epsilon \sigma) \mathrm{e}^{\alpha(r)} r^{2}\left(\frac{d B(r)}{d r}+2 \frac{d \alpha(r)}{d r} B(r)+\frac{4 B(r)}{r}\right),
$$

which corresponds to (77).

[1] A. R. Brown, D. A. Roberts, L. Susskind, B. Swingle, and Y. Zhao, Phys. Rev. Lett. 116, 191301 (2016).

[2] A. R. Brown, D. A. Roberts, L. Susskind, B. Swingle, and Y. Zhao, Phys. Rev. D 93, 086006 (2016).

[3] L. Susskind, Fortschr. Phys. 64, 24 (2016); 64, 44 (2016); D. Stanford and L. Susskind, Phys. Rev. D 90, 126007 (2014); 64, 49 (2016).

[4] L. Lehner, R. C. Myers, E. Poisson, and R. D. Sorkin, Phys. Rev. D 94, 084046 (2016).

[5] R. G. Cai, S. M. Ruan, S. J. Wang, R. Q. Yang, and R. H. Peng, J. High Energy Phys. 09 (2016) 161.

[6] W. D. Guo, S. W. Wei, Y. Y. Li, and Y. X. Liu, Eur. Phys. J. C 77, 904 (2017).

[7] P. Wang, H. Yang, and S. Ying, Phys. Rev. D 96, 046007 (2017).

[8] T. Clifton and J. D. Barrow, Phys. Rev. D 72, 103005 (2005); 90, 029902(E) (2014).

[9] T. Multamaki and I. Vilja, Phys. Rev. D 74, 064022 (2006).

[10] S. Capozziello, A. Stabile, and A. Troisi, Classical Quantum Gravity 25, 085004 (2008).

[11] R. Saffari and S. Rahvar, Mod. Phys. Lett. A 24, 305 (2009).

[12] L. Sebastiani and S. Zerbini, Eur. Phys. J. C 71, 1591 (2011).
[13] G. Cognola, E. Elizalde, L. Sebastiani, and S. Zerbini, Phys. Rev. D 86, 104046 (2012).

[14] M. Visser, Phys. Rev. D 48, 5697 (1993).

[15] S. Deser and B. Tekin, Phys. Rev. D 67, 084009 (2003); 75, 084032 (2007).

[16] G. Abreu and M. Visser, Phys. Rev. Lett. 105, 041302 (2010).

[17] R. G. Cai, L. M. Cao, Y. P. Hu, and N. Ohta, Phys. Rev. D 80, 104016 (2009).

[18] S. Chakraborty and T. Padmanabhan, Phys. Rev. D 92, 104011 (2015).

[19] S. Chakraborty, J. High Energy Phys. 08 (2015) 029.

[20] G. Cognola, O. Gorbunova, L. Sebastiani, and S. Zerbini, Phys. Rev. D 84, 023515 (2011).

[21] J. B. Pendry, J. Phys. A 16, 2161 (1983).

[22] T. M. Cover and J. A. Thomas, Elements of Information Theory (John Wiley \& Sons, New York, 1991).

[23] J. D. Bekenstein and A. E. Mayo, Gen. Relativ. Gravit. 33, 2095 (2001).

[24] D. Carmi, S. Chapman, H. Marrochio, R. C. Myers, and S. Sugishita, J. High Energy Phys. 11 (2017) 188.

[25] S. Nojiri and S. D. Odintsov, Int. J. Geom. Methods Mod. Phys. 04, 115 (2007); Phys. Rep. 505, 59 (2011).

[26] T. P. Sotiriou and V. Faraoni, Rev. Mod. Phys. 82, 451 (2010). 
[27] A. De Felice and S. Tsujikawa, Living Rev. Relativity 13, 3 (2010).

[28] G. W. Gibbons and S. W. Hawking, Phys. Rev. D 15, 2752 (1977).

[29] I. L. Buchbinder and S. L. Lyakhovich, Classical Quantum Gravity 4, 1487 (1987).

[30] M. S. Madsen and J. D. Barrow, Nucl. Phys. B323, 242 (1989).

[31] L. Fatibene, M. Ferraris, and M. Francaviglia, Int. J. Geom. Methods Mod. Phys. 02, 373 (2005).

[32] M. Francaviglia and M. Raiteri, Classical Quantum Gravity 21, 3459 (2004).

[33] A. Guarnizo, L. Castaneda, and J. M. Tejeiro, Gen. Relativ. Gravit. 42, 2713 (2010).

[34] N. Deruelle, M. Sasaki, and Y. Sendouda, Prog. Theor. Phys. 119, 237 (2008).

[35] N. Deruelle, Y. Sendouda, and A. Youssef, Phys. Rev. D 80, 084032 (2009).

[36] N. Deruelle, M. Sasaki, Y. Sendouda, and D. Yamauchi, Prog. Theor. Phys. 123, 169 (2010).

[37] S. W. Hawking and C. J. Hunter, Classical Quantum Gravity 13, 2735 (1996).

[38] A. Vilenkin, Phys. Rev. D 32, 2511 (1985).

[39] S. Capozziello, Int. J. Mod. Phys. D 11, 483 (2002).

[40] G. Cognola, M. Gastaldi, and S. Zerbini, Int. J. Theor. Phys. 47, 898 (2008).

[41] D. R. Brill, J. Louko, and P. Peldan, Phys. Rev. D 56, 3600 (1997).
[42] G. Cognola, M. Rinaldi, L. Vanzo, and S. Zerbini, Phys. Rev. D 91, 104004 (2015).

[43] L. Vanzo, Phys. Rev. D 56, 6475 (1997).

[44] R. M. Wald, Phys. Rev. D 48, R3427 (1993).

[45] S. W. Hawking, Nature (London) 248, 30 (1974); Commun. Math. Phys. 43, 199 (1975)

[46] M. K. Parikh and F. Wilczek, Phys. Rev. Lett. 85, 5042 (2000).

[47] R. Di Criscienzo, S. A. Hayward, M. Nadalini, L. Vanzo, and S. Zerbini, Classical Quantum Gravity 27, 015006 (2010).

[48] M. Alishahiha, A. Faraji Astaneh, A. Naseh, and M. H. Vahidinia, J. High Energy Phys. 05 (2017) 009.

[49] S. A. Hayward, Classical Quantum Gravity 15, 3147 (1998); S. A. Hayward, R. Di Criscienzo, L. Vanzo, M. Nadalini, and S. Zerbini, Classical Quantum Gravity 26, 062001 (2009).

[50] H. Kodama, Prog. Theor. Phys. 63, 1217 (1980).

[51] S. Deser, O. Sarioglu, and B. Tekin, Gen. Relativ. Gravit. 40, 1 (2008).

[52] E. Bellini, R. Di Criscienzo, L. Sebastiani, and S. Zerbini, Entropy 12, 2186 (2010).

[53] W. T. Zaumen, Nature (London) 247, 530 (1974).

[54] G. W. Gibbons, Commun. Math. Phys. 44, 245 (1975).

[55] B. Carter, Phys. Rev. Lett. 33, 558 (1974).

[56] D. N. Page, Phys. Rev. D 14, 3260 (1976).

[57] D. N. Page, Phys. Rev. D 13, 198 (1976). 